1 Title: Landmark navigation in a mantis shrimp

2 Authors: Rickesh N. Patel ${ }^{1 *}$, Thomas W. Cronin ${ }^{1}$

3 Affiliations: ${ }^{1}$ The University of Maryland, Baltimore County.

4 UMBC Department of Biological Sciences, 1000 Hilltop Circle, Baltimore, Maryland 21250

$5 \quad *$ Correspondence to: rickp1@umbc.edu

\title{
7 Summary:
}

Mantis shrimp are predatory crustaceans that commonly occupy burrows in shallow,

9 tropical waters worldwide. Most of these animals inhabit structurally complex, benthic

10 environments where many potential landmarks are available. Mantis shrimp of the species

11 Neogonodactylus oerstedii return to their burrows between foraging excursions using path

12 integration, a vector-based navigational strategy that is prone to accumulated error. Here we

13 show that $N$. oerstedii can navigate using landmarks in parallel with their path integration

14 system, offseting error generated when navigating using solely path integration. We also report

15 that when the path integration and landmark navigation systems are placed in conflict, $N$.

16 oerstedii will orient using either system or even switch systems enroute. How they make the

17 decision to trust one navigational system over another is unclear. These findings add to our

18 understanding of the refined navigational toolkit $N$. oerstedii relies upon to efficiently navigate

19 back to its burrow, complementing its robust, yet error prone, path integration system with

20 landmark guidance. 
22 Key Words: navigation, path integration, landmark navigation, visual guidance, mantis shrimp,

23 stomatopod, homing, marine biology

Introduction:

Stomatopods, better known as mantis shrimp, are benthic crustaceans renowned for their

27 ballistic strikes and complex visual systems. As adults, most mantis shrimp species reside in

28 shallow tropical marine waters, environments that are often structurally varied and therefore contain many potential visual landmarks [1]. In these environments, mantis shrimp typically occupy small holes or crevices for use as burrows, where they reside concealed for most of the day. During foraging, many stomatopod species leave the safety of their burrows for extended excursions, where they become vulnerable to predation [2-5]. Returning to the burrow efficiently is critical to minimize predation risk and to also reduce the chance that the vacated burrow will be claimed by another animal.

Mantis shrimp of the species Neogonodactylus oerstedii employ path integration to efficiently navigate back to their burrows between foraging bouts [5]. During path integration, an

37 animal monitors the distances it travels in various directions from a reference point (usually home) using a biological compass and odometer. From this information, a home vector (the most

39 direct path back to the reference point) is continuously calculated, allowing the animal to return to its original location [6-8]. As animals update their home vectors during excursions, small

41 errors in odometric and orientation measurements are made. Over the course of an animal's

42 travel, these small errors accumulate in its path integrator. Therefore, with longer outward paths, 43 increased errors of home vectors are expected [7, 9]. Path integration using idiothetic cues (those 
44 informed by stimuli anchored internal to the body) are particularly prone to accumulated error.

45 As theory suggests, path integration in N. oerstedii is prone to this accumulated error [10]. To

46 reduce this error, many path-integrators use landmarks to accurately pinpoint their goal $[9,11$ -

47 14]. We hypothesized that in addition to path integration, $N$. oerstedii uses landmarks when

48 available during navigation. The benthic habitats $N$. oerstedii occupy are structurally complex

49 with an abundance of sponges, coral, rock, and seagrass to serve as potential visual landmarks

50 (Fig. 1). Using landmarks during navigation would allow N. oerstedii to correct for error

51 accumulated while path-integrating during foraging paths away from the burrow.

53 Results:

\section{Neogonodactylus oerstedii uses landmarks during navigation}

We placed $N$. oerstedii individuals in relatively featureless circular arenas filled with

56 sand and sea water in a glass-roofed greenhouse. Vertical burrows were buried in the sand so that

57 they were hidden from view when experimental animals were away. Snail shells stuffed with

58 small pieces of shrimp were placed at one of two fixed locations approximately $70 \mathrm{~cm}$ from the

59 location of the burrow in the arena (Fig. 2A). Foraging paths to and from the location of the food

60 were video recorded from above.

As described by Patel and Cronin (2020a,b) [5,10], we observed that animals would make

62 tortuous paths away from the burrow until they located the food placed in the arena. After

63 animals located the food, they would usually execute a fairly direct home vector towards the

64 burrow. If the burrow was not found using the home vector, animals would initiate a stereotyped 65 search behavior (Fig. 2C and Extended Data Video 1). 
To determine if $N$. oerstedii use landmarks during homeward navigation when available,

67 a 2-cm diameter, 8-cm tall vertical cylinder with alternating 1-cm thick horizontal black and white stripes was placed adjacent to the burrow to serve as a landmark. Stripe cycles of the

69 landmark would appear to span approximately 0.8 cycles/degree at the location of the food, approximately twice the visual resolving limit of Gonodactylus chiragra [15], a closely related mantis shrimp that can be slightly larger than N. oerstedii. Trials with the landmark present were compared to the results of previous experiments in which the landmark was absent [5]. absence ( $\mathrm{P}<0.05$; Fig. 2C-D and 3, and Extended Data Videos 1 and 2), supporting the hypothesis that $N$. oerstedii uses landmarks during navigation. This was primarily due to the virtual elimination of stereotyped search behaviors at the ends of homeward paths in the presence

77 of the landmark. Instead, short directed searches for the burrow around the landmark were

78 observed. Return trips were initially oriented similarly between the two groups (Groups were oriented: $\mathrm{P}<0.001$ for both groups; Orientations were not significantly different between groups: $\mathrm{P}>0.5$; All statistical outcomes are presented in Tables 1-3). However, during trials in the presence of the landmark, individuals appeared to correct for their initial homeward error

82 over the course of the homeward path $(\mathrm{P}<0.05)$, in contrast to what we observed in the absence

83 of the landmark $(\mathrm{P}>0.5$; Fig. 2D-F). These results indicate that in the presence of a landmark, $N$.

84 oerstedii uses both path integration and landmark navigation to navigate back to its burrow. 
In light of the above results, we were interested in the confidence $N$. oerstedii places in its

89 landmark navigation system when it is in conflict with its path integrator. In order to create this

90 situation, homeward paths were observed when a landmark adjacent to the burrow was displaced

91 to a new location in the arena while experimental individuals were away foraging. The landmark

92 remained at roughly the same distance from the food location both before and after displacement.

93 If $N$. oerstedii navigates using landmarks and trusts a landmark's location over the location

94 designated by its path integrator when homing, animals should orient towards the displaced

95 landmark rather than the burrow's location (Fig. 2B).

Homeward paths were less direct $(\mathrm{P}<0.05$; Fig. 3) and were differently oriented $(\mathrm{P}<$

$97 \quad 0.05$; Fig. 2D-F) when landmarks were displaced compared to when they were left in place, further supporting the hypothesis that $N$. oerstedii navigate using landmarks. Some individuals oriented towards the displaced landmark while others ignored the displaced landmark, orienting towards the burrow (Fig. 2C and Extended Data Videos 3 and 4). Several individuals initially oriented towards the displaced landmark, but broke away from their initial trajectories during their homeward paths, orienting towards the burrow instead (Fig. 2D). Overall, however, differences observed between initial path orientations and the orientations of homeward paths at

104 the end of the home vector were not statistically significant when the landmark was displaced (P $105=0.36$; Fig. 2E-F). One individual initially oriented its homeward path towards the landmark, 106 only to turn around and return to the food location before adopting a revised homeward path 107 oriented towards the burrow (Fig. 2D). These observations suggest that the path integrator of $N$. 108 oerstedii is continually updated during foraging, even after homeward paths are initiated. 
111 completely, orienting towards the burrow. These results demonstrate that $N$. oerstedii must make

112 decisions when the navigational strategies it relies on are in conflict and raise the question of

113 how these decisions are made.

Due to errors inherit in path integration, $N$. oerstedii exhibit growing home vector errors

115 with increased outward path lengths [10]. When the landmark was displaced, individuals may

116 have evaluated this accumulated error during foraging, choosing to trust the position of the

117 landmark when the accumulated error of the path integrator was high (i.e. confidence in the path

118 integrator was low). However, we found that the orientations of homeward paths during

119 landmark displacement experiments were not significantly correlated with the outward path

120 lengths from the burrow to the food location $(\mathrm{P}=0.16$; Fig. 4A); nonetheless, the effect size of

121 this relationship was fairly strong $(r=-0.48)$, suggesting this hypothesis should not be

122 completely discounted.

124 integration and visual landmarks. In experiments with these ants, when their path integrators are 125 placed conflict with their surrounding landmark panorama, displaced desert ants will orient

126 toward either the location indicated by their path integrator or toward a local landmark array

127 depending on their distance from their nest, not on the error accumulated in their path

128 integrators. These ants will orient using their home vectors, ignoring local landmarks, when

129 displaced from at a distance greater than three meters from their nest; however, they will orient

130 using the local landmark array when displaced from near the nest. When displaced from a

131 distance of one meter from their nest, desert ants will orient with a mean vector not clearly

132 directed at either their home vectors derived from path integration or the local landmark

133 panorama, but somewhere in between [16]. Interestingly, orientation results of the desert ants 
134 displaced from roughly one meter from the nest are similar to those of $N$. oerstedii during the

135 landmark displacement experiments described above. Stomatopods in those experiments were

136 around $0.7 \mathrm{~m}$ from their burrows when initiating their homeward paths (Fig. 3E). These

137 observations suggest that a cue integration mechanism resembling that employed by desert ants

138 may also be present in mantis shrimp.

As an alternative hypothesis to account for the variation observed in homeward paths and the landmark's perceived position may have been at a preference threshold for either of the or the displaced landmark during these trials.

152 displacement of the landmark. To investigate this hypothesis, we measured the orientations of all 153 animals' body axes with respect to the landmark while it was displaced, sampled at a rate of 0.2

154 seconds. We compared the means of these body axis orientations to the orientations of

155 homeward paths and found no correlation $(\mathrm{P}=0.604, \mathrm{r}=0.19$; Fig. 4C). This suggests that either 156 animals did not notice the landmark's displacement or that observing the landmark's 
157 displacement did not influence an animal's decision to determine the burrow's location by using

158 the displaced landmark's position or by using its home vector.

\section{Discussion:}

Our results demonstrate that Neogonodactylus oerstedii uses landmark navigation

together with path integration while navigating back to its burrow while foraging. Landmarks are reliable references which can be used to correct for error accumulated by path integration; this is especially important during idiothetic path integration, which $N$. oerstedii uses when allothetic cues become unreliable [5].

167 home towards. Many other questions about how landmarks may be used by mantis shrimp arise

168 from this work: Can mantis shrimp estimate the relative position of a goal to multiple

169 landmarks? Do stomatopods use a snapshot mechanism like that employed by some insects to 170 learn landmark arrays $[13,17]$ ? Do they possess cognitive maps akin to those thought to exist in

171 mammals [18]? Do mantis shrimp learn to recognize landmarks encountered during foraging

172 routes, exhibiting "trapline foraging"? Further, mantis shrimp are famed for possessing complex

173 color vision, linear polarization vision in two spectral channels, and circular polarization vision

174 [19]. Besides spatial vision alone, do stomatopods use these visual channels to identify

175 landmarks? If so, how?

177 complex reefs to nearly featureless mud flats. Stomatopod species that occupy landmark-rich

178 environments may weigh the importance of landmarks more heavily during navigation than 
179

180

181

182

stomatopods which occupy benthic environments relatively void of landmarks. Further, visual information rapidly attenuates with distance underwater due to extreme scattering of light in water. Therefore, the relative importance of landmark navigation over path integration may differ for mantis shrimp species occupying waters of different depths and turbidities.

Taken together with our previous work on mantis shrimp navigation $[5,10]$, this work offers an opportunity to study the neural basis of navigation, learning, memory, and decision making in stomatopods. Mushroom bodies, centers for arthropod learning and memory, are thought to play a prominent role in landmark learning in insects [20-23]. Prominent hemiellipsoid bodies, homologues of insect mushroom bodies, exist in stomatopod eyestalks [24]. As in insects, these neuropils may be crucial for navigation and landmark learning in mantis shrimp. A separate brain region, the central complex, plays a role in landmark orientation in Drosophila melanogaster. Here, landmark orientation is neurally based in the ellipsoid body of the central complex [25]. Stomatopods themselves possess a highly developed central complex composed of a collection of neuropils anatomically very similar to those found in insects [26]. Investigation of the function of stomatopod brain regions in light of our work may have implications for the evolutionary origins of navigational strategies and the neural architecture of the brain within the ancient Pancrustacean clade, a taxon which includes all insects and crustaceans [27], as well as in other arthropods.

In summary, $N$. oerstedii possesses a robust navigational toolkit on which it relies to efficiently navigate back to its burrow. First, N. oerstedii relies on path integration using multiple redundant compass cues to navigate back to its home [5]. If path integration does not lead $N$. oerstedii directly to its burrow, it relies on a stereotyped search behavior which is scaled to the amount of error it accumulates during its outbound foraging path to locate its nearby lost target 
202 [10]. Finally, the stomatopod will use landmarks, if available, to quickly pinpoint its target,

203 offsetting error accumulated during path integration.

Acknowledgements: We thank N.S. Roberts and J. Park for research assistance.

206

207
Funding: This work was supported by grants from the Air Force Office of Scientific Research under grant number FA9550-18-1-0278 and the University of Maryland Baltimore County.

Author Contributions: R.N.P. designed and conducted all research, analyzed all data, and prepared the manuscript. T.W.C. provided guidance and research support.

Competing Interests: The authors declare no competing financial interests.

Data and Materials Availability: The data that support the findings of this study are available from the corresponding author upon reasonable request. Correspondence and requests for

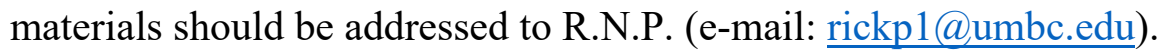




\section{Figures:}
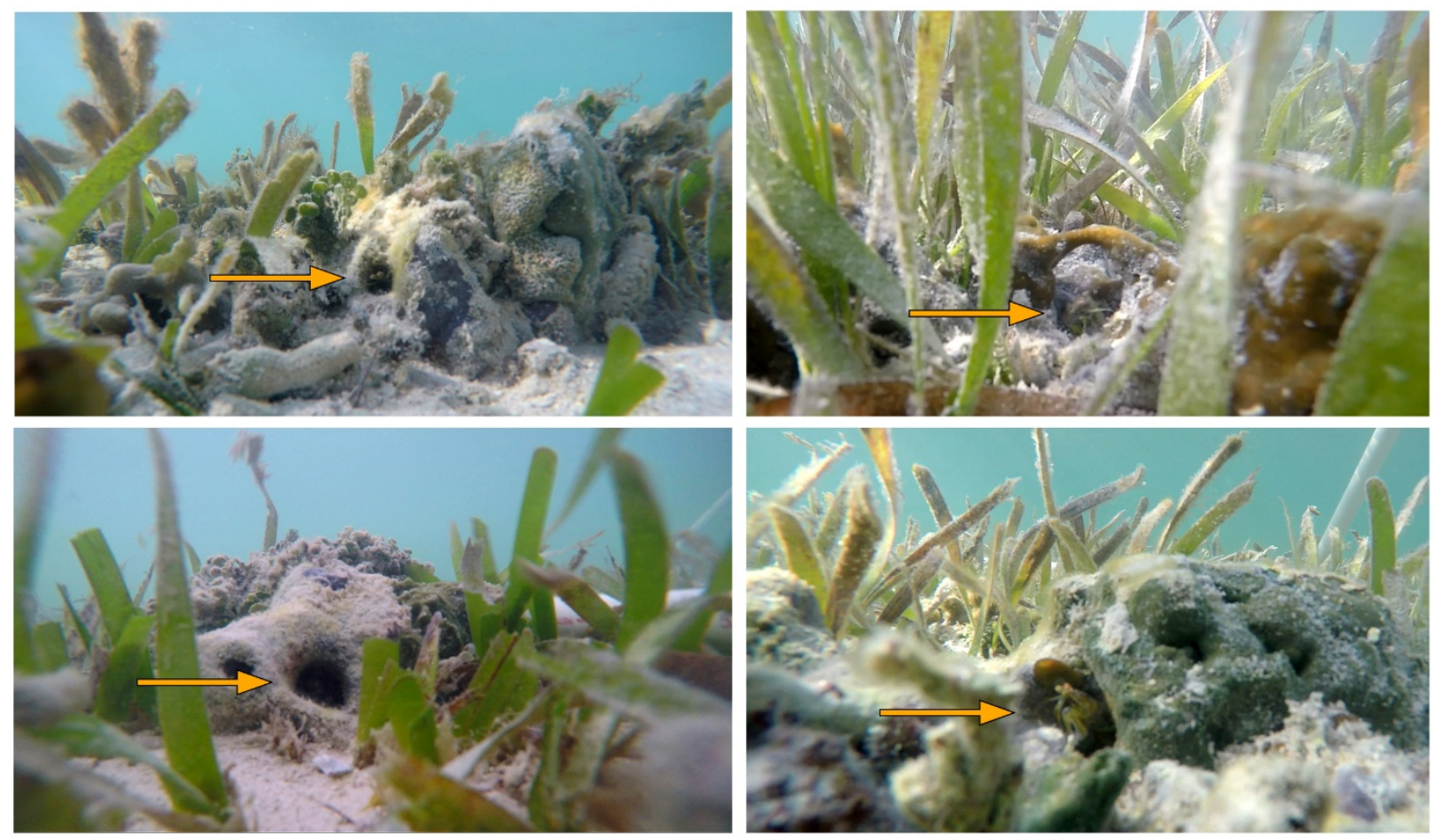

Figure 1. Neogonodactylus oerstedii inhabits shallow waters that offer an abundance of

225 potential landmarks. Burrows are indicated by orange arrows. Note the abundance of potential

226 landmarks, including marine vegetation, sponges, coral fragments, and rock rubble, available in

227 the scenes. Stomatopods can be seen in their burrows in all except the bottom left panel, in which

228 the photograph was taken when the animal had left its home. 
A

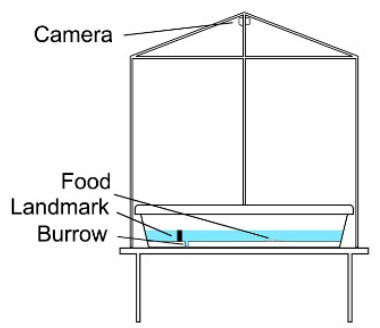

C

Landmark Absent

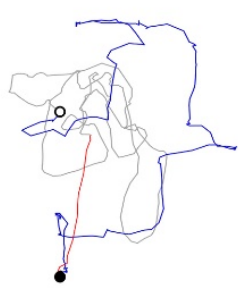

D
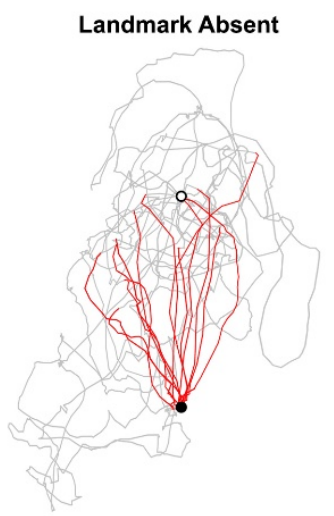

$\mathbf{E}$

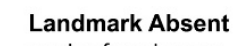
angles from burrow

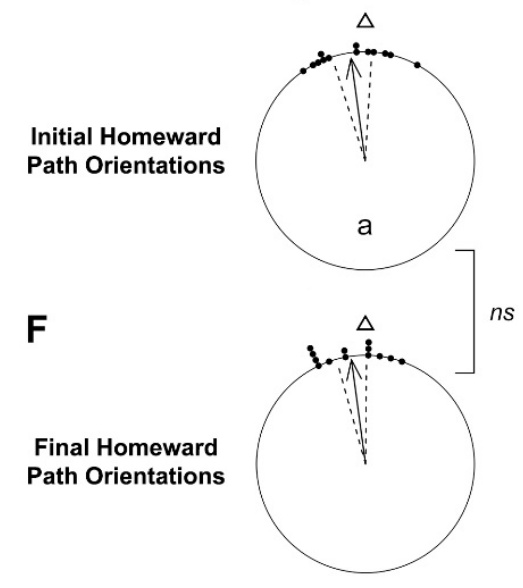

B

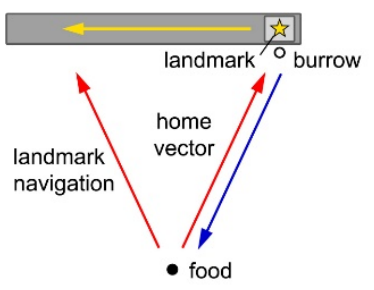

Landmark Displaced
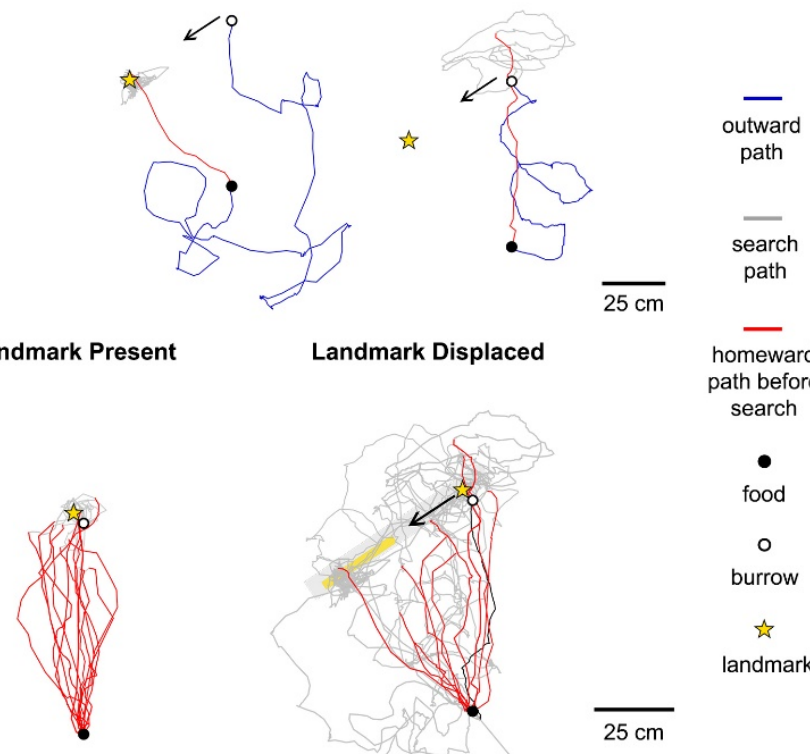

Landmark Present

Landmark Displaced

homeward
path before search

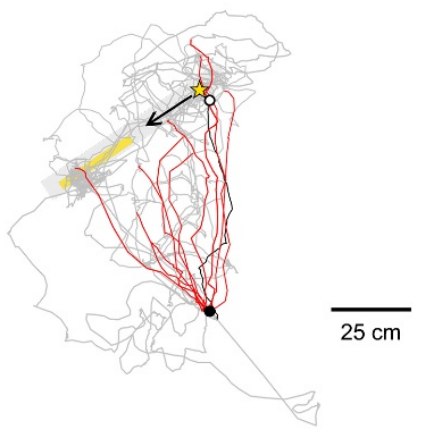

$\bullet$

food

0

burrow

¿

landmark
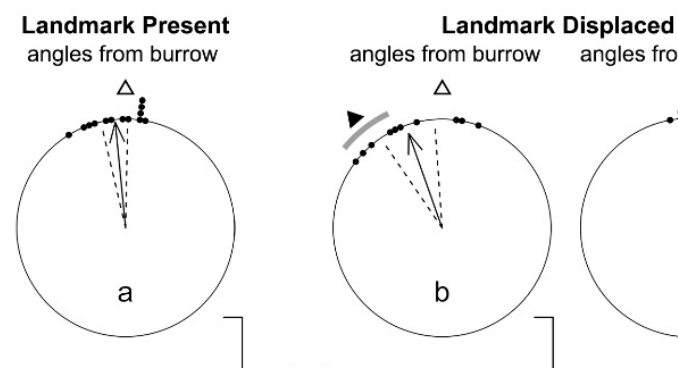

angles from burrow angles from landmark

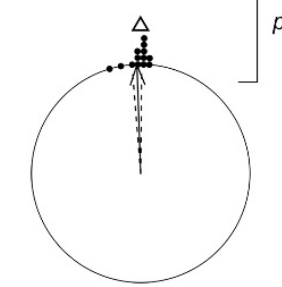

$p<0.05$
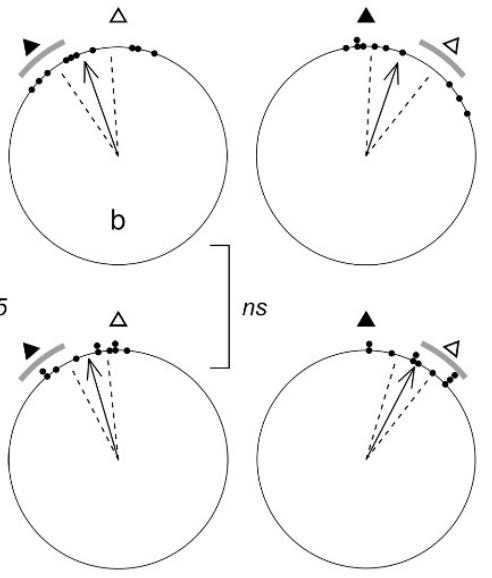

burrow direction landmark direction
foraging. (A) Navigation arenas. Each arena was $150 \mathrm{~cm}$ diameter. A vertical burrow was set 
233 into the base of the arena $30 \mathrm{~cm}$ from the edge of the pool so it was invisible at range (empty 234 circle). A landmark was placed adjacent to the burrow during some experiments (gold-filled 235 star). Food was placed in one of two locations near the center of the pool (filled circles).

236 Behaviors were video recorded from above. (B) Landmark displacement experimental design.

237 Homeward paths were observed when a landmark adjacent to the burrow was displaced to a new

238 location in the arena while experimental individuals were away foraging. (C) Examples of

239 foraging paths from and to the burrow during the three experimental conditions. Blue lines

240 represent outward paths from the burrow while red lines represent homeward paths before search

241 behaviors were initiated. Grey lines represent homeward paths after search behaviors were

242 initiated. Empty and filled circles represent the location of the burrow and food, respectively.

243 Gold-filled stars represent the location of the landmark. Arrows represent paths of landmark

244 displacements. (D) Data from all homeward paths. Lines and filled circles represent the same as

245 in (C). The grey rectangle represents the track along which the landmark was displaced. The gold

246 rectangle marks the range of locations to which the landmark was displaced during landmark

247 displacement trials. The black tracing in the "landmark displaced" group marks the homeward

248 path of an individual on its second run which, after orienting its initial homeward path towards

249 the displaced landmark (in red), it returned to the food location and oriented towards the burrow

250 (in black). (E) Orientations of homeward paths at one-third the beeline distance from the location

251 of the food to the burrow (initial orientations). Each point along the circumference of the circular

252 plot represents the orientation of the homeward path of one individual with respect to either the

253 actual position of the burrow (empty triangle) or displaced landmark's position (filled triangle).

254 Grey arcs in the "Landmark Displaced" orientation plots represent the range of the directions of

255 the either the displaced landmark or the burrow from at the location of the food. Arrows in each 
256 plot represent mean vectors, where arrow angles represent vector angles and arrow lengths

257 represents the strength of orientation $(\bar{R})$. Dashed lines represent $95 \%$ confidence intervals.

258 Different letters within orientation plots denote a significant difference between groups $(\mathrm{p}<0.05)$.

259 "Landmark Absent" data were obtained from Patel and Cronin (2020a) [5]. (F) Homeward path

260 orientations of groups same as in (E) measured immediately before search behaviors were

261 initiated (final orientations).

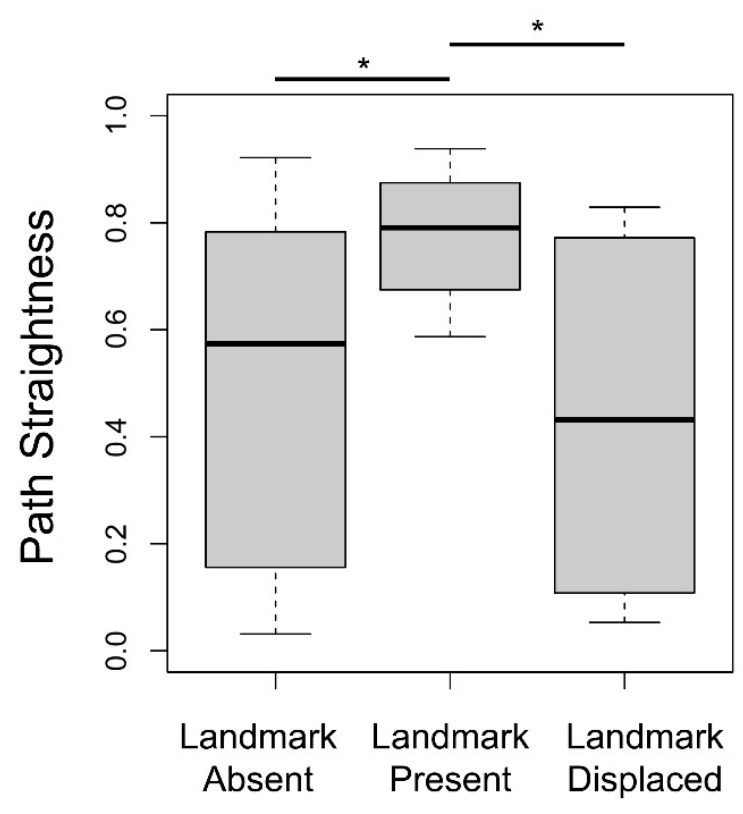

Figure 3. Homeward paths were more direct when a fixed landmark was present during navigation than when the landmark was absent or displaced to a new location in the arena during foraging. Straightness of homeward paths from the location of food to the burrow during

267 trials when the landmark was present, absent, and displaced. Larger path straightness values

268 indicate straighter paths with a value of one being a completely straight path from the food

269 location to the burrow (a beeline path). Bars represent medians, boxes indicate lower and upper

270 quartiles, and whiskers show sample minima and maxima. Asterisks indicate significant 
271 differences in path straightness between groups $(\mathrm{P} \leq 0.05$; Landmark Absent: $\mathrm{n}=13$, Landmark

272 Present: $\mathrm{n}=13$, Landmark Displaced: $\mathrm{n}=10$ ).

273

274

275

276

277

278

279

280

281

282

283

284

285

286

287

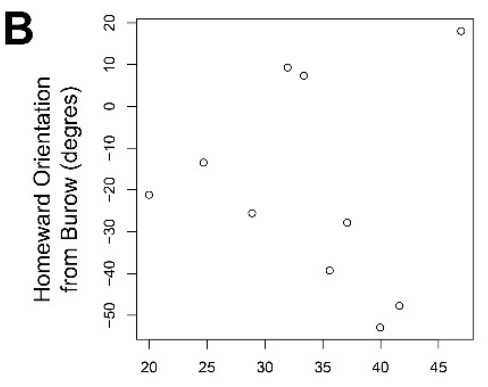

Angular Distance Landmark was Displaced (degrees)

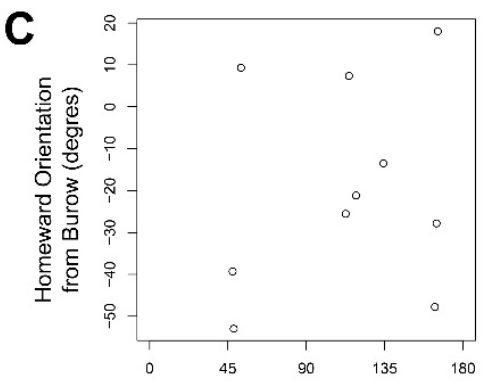

Mean Body Axis from Landmark (degrees)

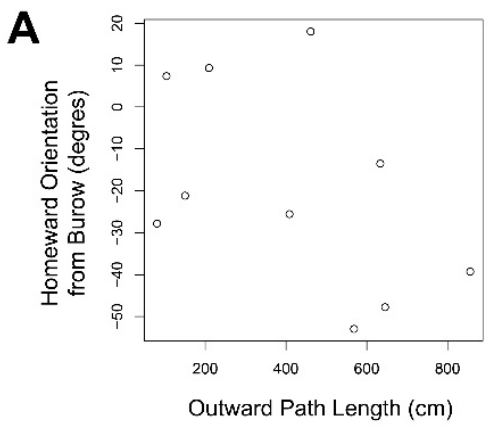

Figure 4. It is unclear why $N$. oerstedii chose to trust either the landmark or the home

vector while navigating during landmark displacement experiments. (A) The orientations of

homeward paths when the landmark was displaced was not significantly correlated with the

length of outward paths from the burrow to the location of food $(\mathrm{P}=0.16, \mathrm{n}=10, \mathrm{r}=-0.48)$. (B)

The orientations of homeward paths when the landmark was displaced was not correlated with

the angular distance of landmark displacement along the track when viewed from the location of

the food $(\mathrm{P}=0.92, \mathrm{n}=10, \mathrm{r}=-0.04)$. (C) Homeward path orientations were not correlated with

body axis orientations of animals with respect to the landmark during its displacement $(\mathrm{P}=$

$0.604, \mathrm{n}=10, \mathrm{r}=0.19)$. Each point represents the mean body axis orientation of an individual

with respect to the landmark measured at a sampling rate of 0.2 seconds during the landmark's

displacement. 
Tables:

Table 1: Statistical outcomes of orientation analyses for all experimental groups.

Orientations of homeward paths were measured relative to the burrow at one-third the beeline uniformity with Holm-Bonferroni multiple testing corrections were used to determine if groups were oriented. Data from this table can be viewed in Figure 2E and F.

\begin{tabular}{|l|l|l|l|l|l|}
\hline Experiment & $\begin{array}{l}\text { P-value } \\
\text { (uncorrected) }\end{array}$ & $\begin{array}{l}\text { Holm- } \\
\text { Bonferroni } \\
\text { (corrected P- } \\
\text { value) }\end{array}$ & $\mathbf{n}$ & $\overline{\boldsymbol{R}}$ & $\begin{array}{l}\text { Mean Vector } \\
\text { Orientation } \pm \\
\text { S.E.M. }\end{array}$ \\
\hline Landmark Absent (Initial) & $<0.0001$ & $<0.001$ & 13 & 0.949 & $354.4^{\circ} \pm 3.76^{\circ}$ \\
\hline Landmark Present (Initial) & $<0.0001$ & $<0.001$ & 13 & 0.974 & $352.2^{\circ} \pm 5.36^{\circ}$ \\
\hline $\begin{array}{l}\text { Landmark Displaced (with respect } \\
\text { to burrow position; Initial) }\end{array}$ & $<0.0001$ & $<0.001$ & 10 & 0.920 & $340.54^{\circ} \pm 7.76^{\circ}$ \\
\hline $\begin{array}{l}\text { Landmark Displaced (with respect } \\
\text { to landmark position; Initial) }\end{array}$ & $<0.0001$ & $<0.001$ & 10 & 0.894 & $18.79^{\circ} \pm 8.93^{\circ}$ \\
\hline Landmark Absent (Final) & $<0.0001$ & $<0.001$ & 13 & 0.966 & $352.32^{\circ} \pm 4.31^{\circ}$ \\
\hline Landmark Present (Final) & $<0.0001$ & $<0.001$ & 13 & 0.996 & $358.03^{\circ} \pm 1.47^{\circ}$ \\
\hline $\begin{array}{l}\text { Landmark Displaced (with respect } \\
\text { to burrow position; Final) }\end{array}$ & $<0.0001$ & $<0.001$ & 10 & 0.960 & $343.73^{\circ} \pm 5.44^{\circ}$ \\
\hline $\begin{array}{l}\text { Landmark Displaced (with respect } \\
\text { to landmark position; Final) }\end{array}$ & $<0.0001$ & $<0.001$ & 10 & 0.956 & $27.54^{\circ} \pm 5.74^{\circ}$ \\
\hline
\end{tabular}

Table 2: Summary of homogeneity of means circular statistical tests for orientation data.

301 Comparisons of orientation groups in rows without an asterisk were analyzed using a Watson-

302 Wheeler Test of Homogeneity of Means (test statistic is F). Comparisons of groups in rows with an asterisk $\left(^{*}\right)$ were analyzed using a non-parametric Watson's Two-Sample Test of 
Homogeneity (test statistic is $\mathrm{U}^{2}$ ) since they did not adhere to the assumptions of a Watsonfrom this table can be viewed in Figure $2 \mathrm{E}$ and $\mathrm{F}$.

\begin{tabular}{|l|l|l|l|}
\hline Experiment & P-value & $\begin{array}{l}\text { Holm- } \\
\text { Bonferroni }\end{array}$ & $\begin{array}{l}\text { Test } \\
\text { Statistic }\end{array}$ \\
\hline $\begin{array}{l}\text { Landmark Absent (Initial) vs Landmark } \\
\text { Present (Initial) }\end{array}$ & 0.7355 & 0.7355 & 0.1168 \\
\hline $\begin{array}{l}\text { Landmark Present (Initial) vs Landmark } \\
\text { Displaced (with respect to burrow; Initial)* }\end{array}$ & $<0.02$ & $<0.04$ & 0.2227 \\
\hline Landmark Absent: Initial vs Final & 0.9827 & 1 & 0.000048 \\
\hline Landmark Present: Initial vs Final* & $<0.005$ & $<0.015$ & 0.3373 \\
\hline Landmark Displaced: Initial vs Final & 0.7414 & 0.7414 & 0.11234 \\
\hline
\end{tabular}

311 Since the "landmark displaced" group did not adhere to the requirements of a T-test, the row

312 with an asterisk (*) was analyzed using a non-parametric paired Wilcoxon Signed-Rank Test

313 (test statistic is V). The straightness of paths from groups within each comparison were

314 significantly different from one another $(\mathrm{P}<0.05)$. The data from this table can be viewed in

315 Figure 3.

\begin{tabular}{|l|l|l|l|}
\hline Experiment & P-value & Holm-Bonferroni & Test Statistic \\
\hline Landmark Absent vs Landmark Present & 0.0216 & 0.0432 & 2.64 \\
\hline Landmark Present vs Landmark Displaced* & 0.027 & 0.0432 & 49 \\
\hline
\end{tabular}


Materials and Methods:

320

321

323

324

325

326

327

328

329

330

331

332

333

334

335

336

337

338

339

340

\section{$\underline{\text { Animal Care }}$}

Individual Neogonodactylus oerstedii collected in the Florida Keys, USA were shipped to the University of Maryland, Baltimore County (UMBC). Animals were housed individually in 30 parts per thousand (ppt) sea water at room temperature under a 12:12 light:dark cycle. Animals were fed whiteleg shrimp, Litopenaeus vannamei, once per week. Data were collected from 13 individuals ( 5 male and 8 female). All individuals were between 30 and $50 \mathrm{~mm}$ long from the rostrum to the tip of the telson.

\section{Experimental Apparatuses}

Four relatively featureless, circular navigation arenas were constructed from $1.5 \mathrm{~m}-$ diameter plastic wading pools that were filled with pool filter sand and artificial seawater (30 ppt; Fig. 2A). Arenas were placed in a glass-roofed greenhouse on the UMBC campus. The spectral transmittance of light through the greenhouse glass was nearly constant for all wavelengths, excluding the deep-UV-wavelength range (280 to $350 \mathrm{~nm}$; Extended Data Fig. 1A). Celestial polarization information was transmitted through the glass roof of the greenhouse (Extended Data Fig. 1B-D). Vertical burrows created from $2 \mathrm{~cm}$ outer-diameter PVC pipes were buried in the sand $30 \mathrm{~cm}$ from the periphery of the arena so that they were hidden from view when experimental animals were foraging. Vertical $2 \mathrm{~cm}$ diameter, $8 \mathrm{~cm}$ high PVC columns with alternating $1 \mathrm{~cm}$ thick black and white horizontal stripes were placed adjacent to the burrows to function as removable landmarks. Stripe cycle widths of the landmarks were approximately 
341 twice the visual resolving limit of Gonodactylus chiragra (0.8 cycles/degree [13]), a closely

342 related mantis shrimp that can be slightly larger than $N$. oerstedii, when viewed from the food

343 location in the arena (a distance of $70 \mathrm{~cm}$ ). Trials were recorded from above using C1 Security

344 Cameras (Foscam Digital Technologies LLC) mounted to tripods placed above the arenas.

345 During landmark displacement experiments, a thin $11 \times 82 \mathrm{~cm}$ acrylic track with a movable

346 platform was placed adjacent to the burrow (Fig. 2B). A landmark identical to the one used in

347 trials in which the landmark was static, was mounted to the movable platform.

\section{Experimental Procedures}

Individual $N$. oerstedii were placed in each arena and were allowed to familiarize

351 themselves to the arena for 24 hours. During familiarization, the striped landmark was placed

352 adjacent to the burrow, marking it during the animals' initial explorations of the arena.

After familiarization, the landmark was either removed for trials in which the landmark was absent or left in place for trials in which the landmark was present. Empty Margarites sp. replaced with a new individual. 
362 platform when animals were foraging away from their burrows. The distance from the food

363 location to the landmark remained relatively constant while the landmark was displaced.

Foraging paths to food locations and from them to the burrow were video recorded from

367 above. In order to differentiate homeward paths from continued arena exploration, paths from the

368 food locations were considered to be homeward paths when they did not deviate more than $90^{\circ}$

369 from their initial trajectories for at least one-third of the beeline distance (the length of the

370 straightest path) from the food location to the burrow. From these homeward paths, search

371 behaviors were determined to be initiated when an animal turned more than $90^{\circ}$ from its initial

372 trajectory.

Paths were traced at a sampling interval of 0.2 seconds using the MTrackJ plugin [28] in

374 ImageJ v1.49 (Broken Symmetry Software), from which the output is given as Cartesian

375 coordinates. From these data, the inbound and outbound path lengths, beeline distances from

376 food to burrow, and inbound and outbound indices of path straightness were calculated, where

377 Path Straightness $=\frac{\text { beeline distance }}{\text { path length }}=\frac{\sqrt{\left(x_{n}-x_{1}\right)^{2}+\left(y_{n}-y_{1}\right)^{2}}}{\sum_{k=1}^{n}\left(\sqrt{\left(x_{k+1}-x_{k}\right)^{2}+\left(y_{k+1}-y_{k}\right)^{2}}\right)}$

$378 \quad n=$ the last coordinate of the path the beeline distance from the food source to the burrow (at which point the orientation of the

381 home vector was usually observed) and at the end of the home vector (when search behaviors

382 were initiated) were recorded using ImageJ. 
We also measured the orientations of the body axes of all animals in respect to the landmark while it was displaced. These body axis orientations were sampled at a rate of 0.2 seconds. From these body axis orientations a mean body axis orientation was calculated for each individual.

Data from the "Landmark Absent" group in this study were taken from the "Not

Manipulated" trials of the greenhouse experiments published in Patel and Cronin (2020a) [5].

All statistical analyses were run on R (v3.3.1, R Core Development Team 2016) with the "CircStats", "circular", "Hmisc", and "boot" plugins. Orientation data were analyzed using the following procedures for circular statistics [29]. All reported mean values for orientation data are circular means. All circular 95\% confidence intervals were calculated by bootstrapping with replacement over 1000 iterations.

As reported in Patel and Cronin (2020a) [5], no significant difference was observed between homeward orientations of males and females during experiments in the absence of a 396 landmark ( $\mathrm{P}>0.5$; Extended Data Fig. 2)), so data from both sexes were pooled for all 397 experiments.

Rayleigh tests of uniformity were used to determine if homeward paths were oriented within a group for all trials. Parametric Watson-Williams tests for homogeneity of means were 400 used to determine if those group orientations were significantly different from one another. The 401 orientations of groups which did not fit the assumptions of the Watson-Williams test were 402 instead compared using the non-parametric Watson's two sample test of homogeneity. These tests were also used to compare differences between initial homeward path orientations 
404 (orientations at one-third the beeline distance from the food to the burrow) and final homeward

405 path orientations (orientations at the initiation of search behaviors) for each group.

406 Homeward path lengths of trials in which the landmark was present were compared to

407 those in which the landmark was absent using a paired T-test. A paired Wilcoxon signed-rank

408 test was used to compare homeward path lengths of trials in which the landmark was static to

409 those in which the landmark was displaced.

$410 \quad$ Pearson's correlation tests were used for all correlative analyses.

411 Holm-Bonferroni multiple testing corrections were used for all tests when applicable. 

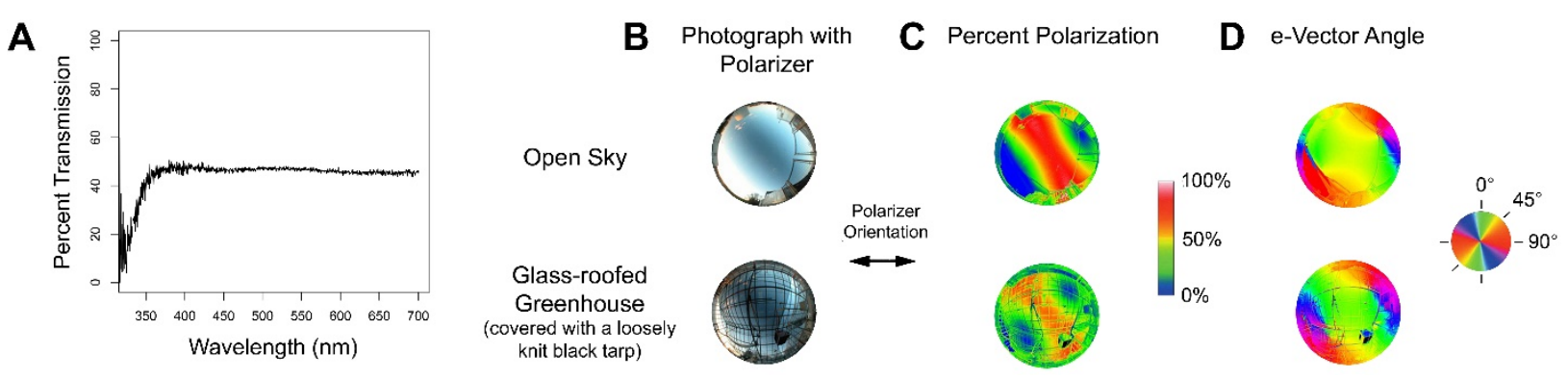

426

Extended Data Figure 1. Photic conditions in the greenhouse where experiments were run.

428

(A) Transmission of irradiance spectra through the glass-roof of the experimental greenhouse near sunset. The spectral transmittance of light through the glass roof of the greenhouse is nearly constant for all wavelengths greater than $\sim 360 \mathrm{~nm}$. (B-D) Celestial polarization patterns are transmitted through the glass roof of the greenhouse. (B) Photographs of the sky at sunset on a day with very few clouds (November 24, 2015) using a fisheye lens and linear polarizer set in the east-west direction (as indicated by the arrow to the right of the photos). Photos were taken

434 inside and outside the glass-roofed greenhouse used for experiments. (C) Percent polarization. 435 Warmer regions in the images indicate higher percent polarization and cooler regions indicate 436 lower percent polarization (see key). (D) e-Vector angle, indicated by the color corresponding 437 the key to the right of the images. From Patel and Cronin (2020a) [5]. 


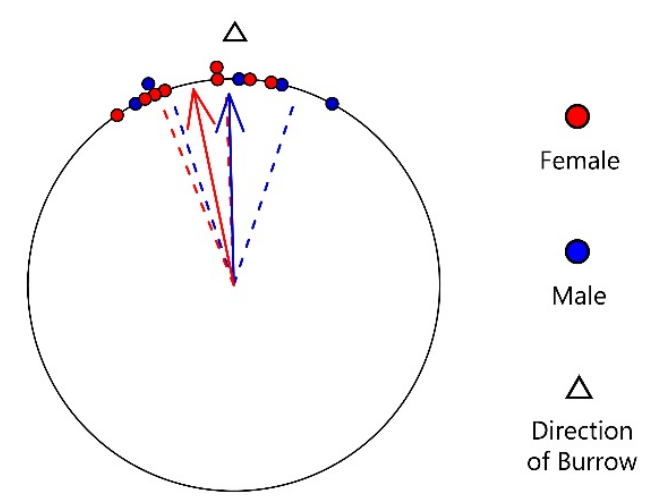

441 Extended Data Figure 2. Male and female $N$. oerstedii orient towards home equally well

442 while foraging. Homeward orientations of male and female individuals during experiments in

443 the greenhouse when animals were not manipulated. Each point along the circumference of the

444 circular plot represents the orientation of the homeward path of one individual with respect to

445 position of the burrow (empty triangle). Blue-filled circles represent males while red-filled

446 circles represent females. Arrows represent mean vectors, where angles of the arrows represent

447 the mean vector angles and arrow lengths represent the strength of orientation in the mean

448 direction $(\bar{R})$. Dashed lines represent $95 \%$ confidence intervals. Males $(\mathrm{n}=5)$ and females $(\mathrm{n}=8)$

449 both exhibited significant orientations ( $p<0.01$ for both groups). No significant difference in

450 orientation was observed between males and females ( $p>0.5)$. From Patel and Cronin (2020a)

451 [5].

452

453

454

455

456

457

458 
Video 1. Foraging behavior of Neogonodactylus oerstedii showing homing in the absence of path is in grey. Filmed at 30 frames per second. Replay speed is indicated in the bottom-right

462 corner of the video.

Video 2. Foraging behavior of Neogonodactylus oerstedii showing homing in the presence of 30 frames per second. Replay speed is in real time.

471 red, and search path is in grey. Filmed at 30 frames per second. Replay speed is indicated in the 472 bottom-right corner of the video.

Video 4. Foraging behavior of Neogonodactylus oerstedii showing homing after a landmark 


\section{References:}

483

484

485

486

487

488

489

490

491

492

493

494

495

496

497

498

499

500

501

502

503

504

1. Reese, E.S. Orientation behavior of butterflyfishes on coral reefs: spatial learning of routine specific landmarks and cognitive maps. Environmental Biology of Fishes. 25, 7986 (1989).

2. Dominguez, J.H. \& Reaka, M. Temporal activity patterns in reef- dwelling stomatopods: a test of alternative hypotheses. Journal of Experimental Marine Biology and Ecology. 117, 47-69 (1988).

3. Basch, L.V. \& Engle, J.M. Aspects of the ecology and behavior of the stomatopod Hemisquilla ensigera californiensis (Gonodactyloidea: Hemisquillidae). Biology of stomatopods 3, 199-212 (1989).

4. Caldwell, R.L., Roderick, G.K. \& Shuster, S.M. Studies of predation by Gonodactylus bredini. Biology of stomatopods 3, 117-131 (1989).

5. Patel, R.N. \& Cronin, T.W. Mantis shrimp navigate home using celestial and idiothetic path integration. Curr. Biol. (in press).

6. Seyfarth, E., Hergenröder, R., Ebbes, H. \& Friedrich, G. Idiothetic Orientation of a Wandering Spider: Compensation of Detours and Estimates of Goal Distance. Behav. Ecol. Sociobiol. 11, 139-148 (1982).

7. Müller, M. \& Wehner, R. Path integration in desert ants, Cataglyphis fortis. Proc. Natl. Acad. Sci. USA 85, 5287-5290 (1988).

8. Seguinot, V., Maurer, R. \& Etienne, A.S. Dead reckoning in a small mammal: the evaluation of distance. J. Comp. Physiol. A 173, 103-113 (1993).

9. Cheung, A., Zhang, S., Stricker, C. \& Srinivasan, M.V. Animal navigation: the difficulty of moving in a straight line. Biol. Cyber. 97, 47-61 (2007). 
10. Patel R.N. and Cronin, T.W. Path integration error and adaptable search behaviors in a mantis shrimp. J. Exp. Biol. (under review).

11. Etienne, A. S. Navigation of a small mammal by dead reckoning and local cues. Curr. Dir. Psychol. Sci. 1, 48-52 (1992).

12. Collett, T. Insect navigation en route to the goal: multiple strategies for the use of landmarks. J. Exp. Biol. 199, 227-235 (1996).

13. Akesson S. \& Wehner R. 1997. Visual snapshot memory of desert ants, Cataglyphis fortis. Proceedings of the Göttingen Neurobiology Conference. 25, 482.

14. Wehner, R. Desert ant navigation: How miniature brains solve complex tasks. Karl von Frisch Lecture. J. Comp. Physiol. A, 189, 579-588 (2003). Comparative Physiology A. 173, 583-594 (1993).

16. Wystrach A., Mangan M., Webb B. \& Mangan M. Optimal cue integration in ants. Proceedings of the Royal Society B: Biological Sciences. 282, 20151484 (2015).

17. Cartwright, B.A. \& Collet, T.S. Landmark learning in bees: experiments and models. J. comp. Physiol. 151, 521-543 (1983). memory task. Experimental Brain Research. 68, 1-27 (1987). vision in mantis shrimps: themes in visible and ultraviolet vision. Phil. Trans. R. Soc. B 369, 20130032 (2014). 
20. Mizunami, M., Weibrecht, J.M. \& Straussfeld, N.J. Mushroom bodies of the cockroach: Their participation in place memory. Journal of Comparative Neurology 402, 520-537 (1998).

21. Heisenberg, M. Mushroom body memoir: From maps to models. Nature Reviews Neuroscience 4, 266-275 (2003).

22. Lutz, C.C. \& Robinson, G.E. Activity-dependent gene expression in honey bee mushroom bodies in response to orientation flight. J. Exp. Biol. 216, 2031-2038 (2013). synaptic organization in the mushroom bodies of the desert ant Cataglyphis fortis. Developmental Neurobiology. 70, 408-423 (2010). mushroom body in a crustacean brain. eLife. 2017;6:e29889 (2017).

25. Seelig, J.D. \& Jayaraman, V. Neural dynamics for landmark orientation and angular path integration. Nature. 251, 186-191 (2015). the stomatopod central complex: functional and phylogenetic implications. Frontiers in Behavioral Neuroscience. 11, 1-18 (2017). Imaging and Spectroscopic Analysis of Living Cells. 504, 183-200 (2012). 